\title{
PERSISTENCE OF RECESSIVE LETHAL GENES ON THE SECOND CHROMOSOME OF DROSOPHILA MELANOGASTER IN EXPERIMENTAL POPULATIONS ${ }^{1)}$
}

\author{
SHIGEMITSU TANO \\ Laboratory of Radiation Genetics, Faculty of Agriculture, \\ The University of Tokyo, Bunkyo-ku, Tokyo 113
}

Received January 20, 1971

\section{INTRODUCTION}

A number of lethal genes on the second chromosome of Drosophila melanogaster were discovered either spontaneously or induced by artificial methods. These lethals would be eliminated from a given population with different speed according to their detrimental effects on viability of their heterozygotes. However, frequencies of lethal heterozygotes are quite different from a population to another population. In some cases, the frequency of lethal genes of $D$. melanogaster was kept unexpectedly at high level for a long time in natural populations (Burdick 1961; Oshima 1969; Watanabe and Oshima 1970) or in cage populations (Spiess et al. 1963; Ytterborn 1968).

In the present report, changes of the frequencies of four kinds of lethal genes in different genetic backgrounds will be presented with artificial populations.

\section{MATERIALS AND METHODS}

Four kinds of recessive lethals on the second chromosome, which showed high fecundity of their heterozygous females in the previous experiments (Tano and Burdick 1965), were used again in the present experiment. Lethal genes in PC 140s, PC 142 and PC 143, were isolated from laboratory populations and PC 207 was isolated from a natural population collected at Erie, Pennsylvania, U.S.A.

Because of the different origin of these lethal genes, the genetic background should be equilibriated to perform the experiment. The genetic backgrounds of these lethal strains were substituted by backcrossing to the homozygous S-2 wild-type strain. This strain originated from W $190 \mathrm{pol}$, was made isogenic by single brother-sister mating for 37 generations and then subsequently has been kept in mass culture. The fourth chromosome of this strain was marked with eye mutant pol/pol (polish). The substitution procedure was shown in Fig. 1. Female flies of the original $C y$ (Curly) balanced lethal strain were mated with male flies of $\mathrm{H} 55\left(F M 4 / Y ; S p / C y ; S b / U b x^{130} ; p o l / p o l\right)$

1) The present study was supported by the Grant No. 96016 from the Ministry of Education. 


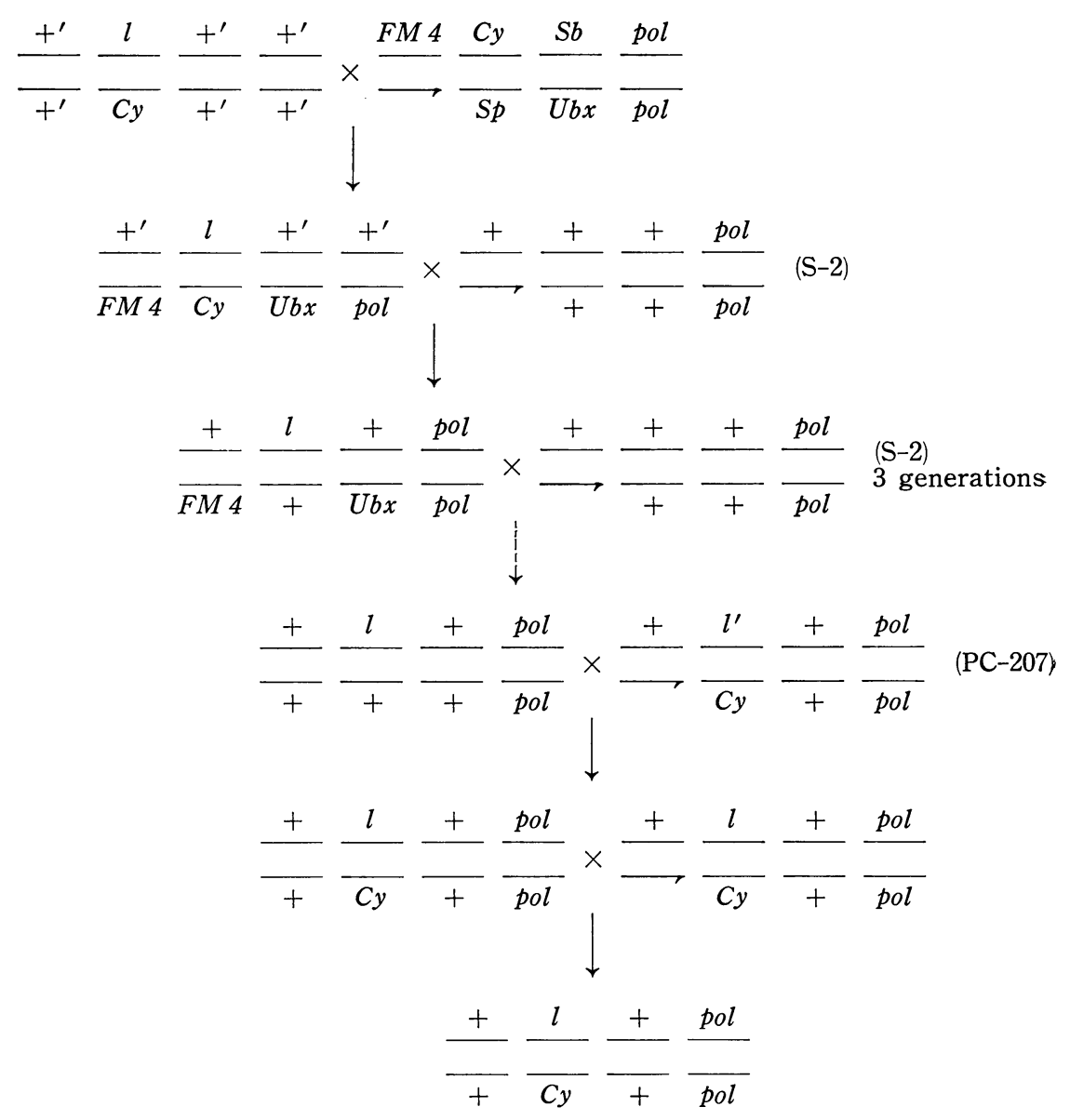

Fig. 1. Mating scheme of background substitution.

strain. Virgin female flies of $F_{1}$ progeny $\left(F M 4 /+^{\prime} ; C y / l ; U b x^{130} /+^{\prime} ; p o l /+^{\prime}\right)$ were mated with male flies having S-2 homozygous chromosomes (five pair matings). In the next generation, virgin female flies $\left(F M 4 /+; l /+; U b x^{130} /+; p o l / p o l\right)$ were backcrossed to homozygous male flies of the S-2 strain. Such backcrossings were repeated for three generations in order to enhance the recombination between the lethal-carrying chromosome and the S-2 second chromosome. After three generations, several female flies having polish eye were crossed with males of PC 207 strain $\left(+/ \mathrm{Y} ; l^{\prime} / C y ;+/+;\right.$ pol/pol) to introduce the $C y$ balancer. The background of PC 207 was previously made co-isogenic with S-2. In the next generation, virgin $C y$ female flies were individually mated with Cy male flies in the same culture to identify the $l / C y$ genotypes and establish the S-2 homozygous background strain. Out of 150 single pair mating cultures, about two or three cultures usually gave rise to balanced $C y$ progenies. The length of unsubstituted chromosome was calculated to be $10 \pm 8.8$ units on both sides of the lethal genes. The heterozygosity of this part was estimated to be about $14 \%$ of the total genome (cf. Tano and Burdick 1965). 

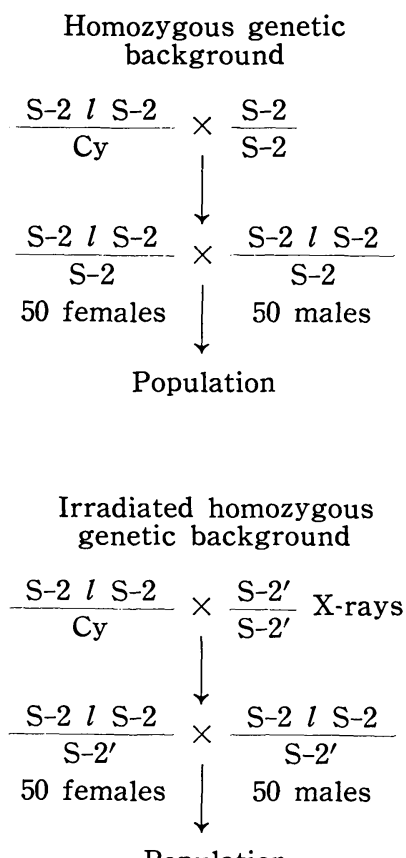

Population

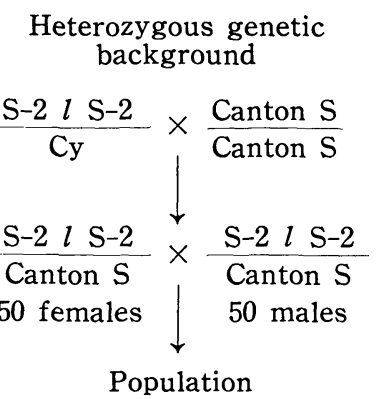

Irradiated heterozygous
genetic background

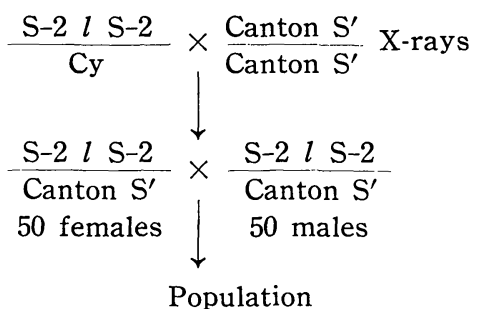

Population

Fig. 2. Procedure of setting up the populations with different genetic background

Virgin female flies carrying these $C y$ balanced lethal gene in the isogenic background were mated with S-2 homozygous male flies to produce $l / \mathrm{S}-2$ progeny. Fifty pairs of females and males of each lethal heterozygote were collected and introduced together into a population cage $(28 \times 38.5 \times 14 \mathrm{~cm})$ to set up populations having the homozygous genetic background (Fig. 2). The population was kept under the environmental conditions of $25^{\circ} \mathrm{C}$ and continuous illumination.

$\mathrm{S}-2$ homozygous male flies were irradiated with $\mathrm{X}$-rays $(250 \mathrm{kVp}, 25 \mathrm{~mA}, 1 \mathrm{~mm} \mathrm{Al}$ filter, $100 \mathrm{R} / \mathrm{min}$ ) and they were mated with virgin female flies of $C y$ balanced lethal strain in order to introduce the irradiated chromosomes into homozygous background. Then, the experimental population was made with non-Cy male and female flies in the next generation.

In the case of heterozygous genetic background, $l / C y$ virgin female flies were mated with male flies of Canton-S strain and the population was set up with 50 pairs of non$C y$ female and male flies of their progeny.

In the case of irradiated heterozygous genetic background, $l / C y$ virgin female flies were mated with irradiated male flies of Canton-S strain and the population was made with 50 pairs of non-Cy female and male flies of their progeny. The mating schemes mentioned above were presented in Fig. 2.

Dosages of X-rays which homozygous background populations have received, were 0kR (Population 0), $1 \mathrm{kR}$ (Population 1), $2 \mathrm{kR}$ (Population 2), $3 \mathrm{kR}$ (Population 3) and $4 \mathrm{kR}$ (Population 4) respectively, and those which heterozygous genetic background populations have received, were $0 \mathrm{kR}$ (Population 10), $1 \mathrm{kR}$ (Population 11), $2 \mathrm{kR}$ (Popu- 
lation 12) and $3 \mathrm{kR}$ (Population 13) respectively.

Two hundreds of males were extracted from each population at certain intervals and divided into four groups. These males were mated individually with 2 virgin females of each tester $l / C y$ strain (a group of 50 males per each lethal strain). $C y$ and non- $C y$ flies emerged from these matings were scored separately. If the male and female flies carry the same lethal gene, the ratio of $C y$ and non-Cy flies in the progeny should be 2 to 1 . The data obtained by such test matings were analyzed using $\chi^{2}$ test.

The value of female fecundity in the previous experiment presented in Table 1 was obtained by the mean egg number layed by lethal heterozygous females in the homozygous and heterozygous genetic backgrounds. These values were compared with that of the appropriate control (Tano and Burdick 1965).

\section{EXPERIMENTAL RESULTS}

As shown in Fig. 3, frequencies of lethal heterozygotes in early generations in the non-irradiated homozygous genetic background have been maintained highly above the the theoretical curve which was calculated as the selection coefficient of the lethal heterozygotes to zero $(s=0)$. The heterotic effect of these lethal genes would be presumed. However, such high frequencies have gradually diminished in later generations except PC 207 lethal heterozygote. The heterotic effect of these lethal genes in the irradiated genetic background was expressed more weakly than that in homozygous genetic background (Population 0). Population 2 was interrupted during the experiment as it was contaminated.

Different effects of heterozygosity of the background on frequencies of the lethal heterozygotes were clearly observed at early generations on heterozygous and irradiated population as shown in Fig. 4. However, frequencies of some lethal heterozygotes, such as PC 207 were considerably higher than theoretical curve in all populations. It was reported in the previous experiment (Tano and Burdick 1965) that heterozygous female flies for each lethal gene had higher fecundity than the isogenic wild-type flies (S-2) in homozygous genetic background. All kinds of lethal heterozygotes have been maintained fairly higher than theoretical level in the homozygous population (top of Fig. 3). When they were introduced into the irradiated homozygous genetic background, they (except PC 207) have decreased faster (see, Population 1,3 and 4).

On the other hand, frequencies of the lethal heterozygote in the heterozygous background decreased more quickly than in the homozygous background as compared with results of Fig. 3 and 4 . Heterotic effects of the lethal gene in early generations were not clearly observed in the heterozygous and its irradiated background. One of the causes illustrating the difference between decreasing patterns, was assumed to be that the heterotic effect of these leathal genes would be covered by heterozygosity of the background in the population. Irradiation did not show the detectable effect for the state of decrease of the lethal heterozygotes in the population. The dosages of $\mathrm{X}$ rays used in the experiment seemed to be not enough to change the genetic background.

When the fecundity of heterozygous female flies for the lethal gene in heterozygous 


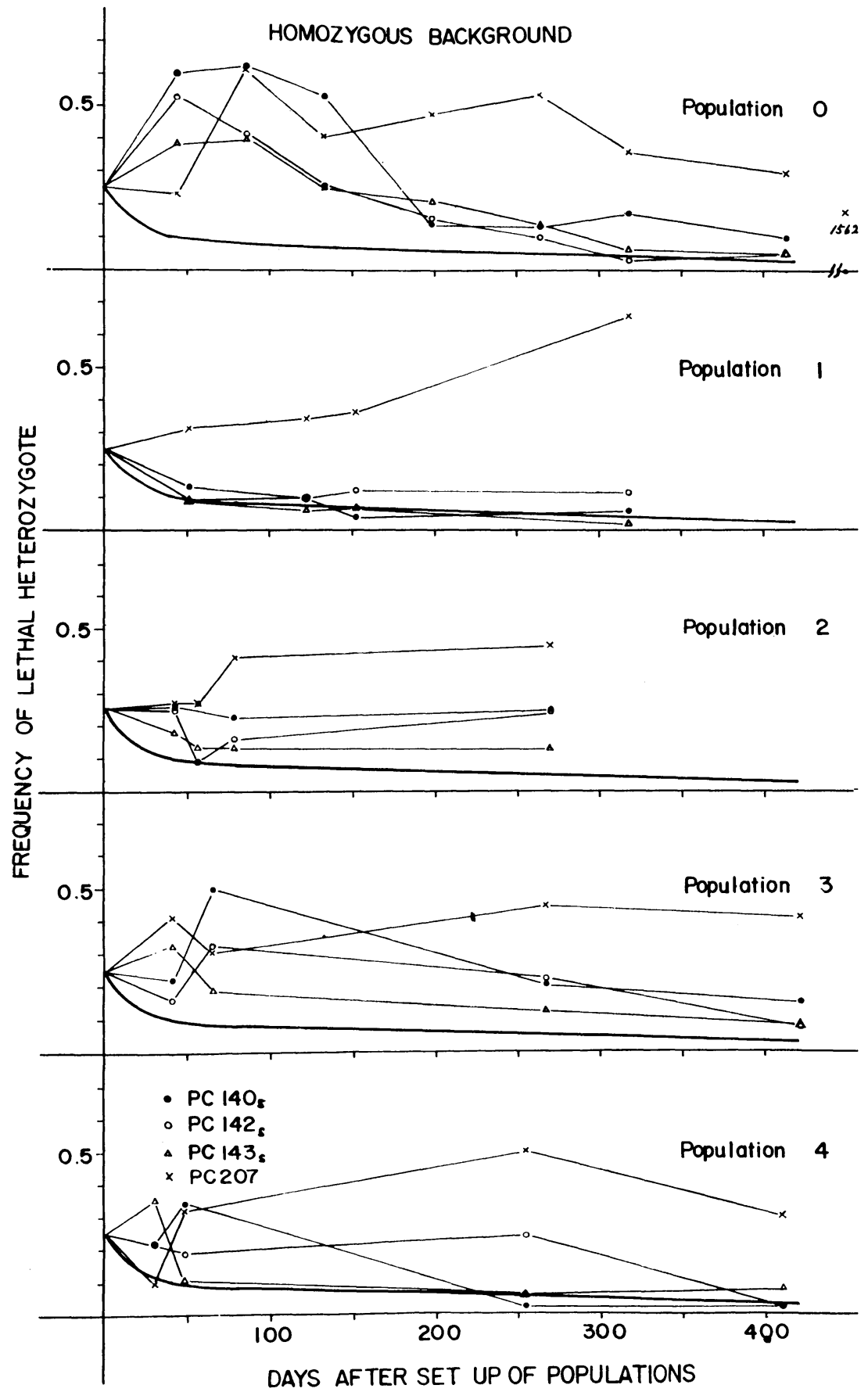

Fig. 3. Changes of frequencies of lethal heterozygotes in populations whose backgrounds were homozygous or irradiated. The following marks represent each lethal heterozygote.
theoretical;
PC 140;
$\bullet \quad \mathrm{PC} 142 \mathrm{~s} ; \mathrm{O}-\mathrm{O}$
$\mathrm{PC} 143_{\mathrm{s}} \Delta-\Delta$

PC 207; $\times-x$ 


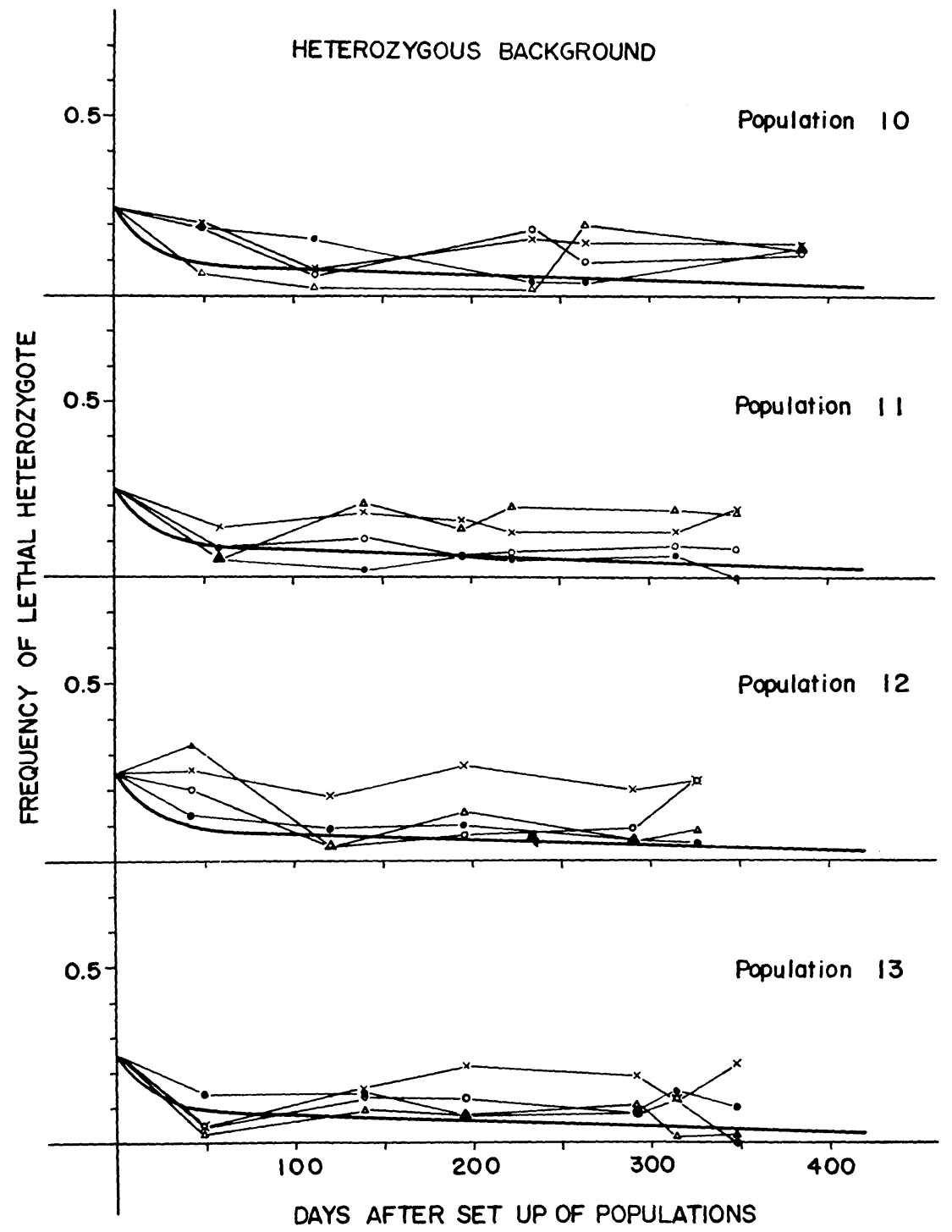

Fig. 4. Changes of frequencise of lethal heterozygotes in populations whose backgrounds were heterozygous or irraidated. The marks are the same in Fig. 3.

genetic background was measured, it showed no significant superiority as compared with that of the normal heterozygotes (Table 1). However, the frequency of some lethals reached to each equilibrium state in populations initiated with heterozygous genetic background in the present experiment. Such different results on equilibrium state from the data presented in Table 1, may be attributable to the method of estimation of female fecundity. The value of fecundity was estimated by the total number of eggs layed by heterozygous females for lethals in $F_{1}$ generation, therefore, the effect of heterozygous chromosomes in $F_{1}$ females canceled the heterotic effect of lethal genes themselves. 
Table 1. Female fecundity (f) of lethal heterozygotes and their estimated equilibrium frequencies (Q)

\begin{tabular}{lccccc}
\hline & \multicolumn{2}{c}{ Homozygous background } & & \multicolumn{2}{c}{ Heterozygous background } \\
\cline { 2 - 3 } \cline { 5 - 6 } Lethal No. & $\mathrm{f}^{*}$ & $\mathrm{Q}$ & & $\mathrm{f}$ & $\mathrm{Q}$ \\
\hline PC $140_{\mathrm{s}}$ & 1.219 & 0.164 & & 1.002 & 0.002 \\
PC $142_{\mathrm{s}}$ & 1.328 & 0.217 & & 1.002 & 0.002 \\
PC $143_{\mathrm{s}}$ & 1.378 & 0.237 & & 0.974 & 0.000 \\
PC 207 & 1.648 & 0.318 & 0.960 & 0.000 \\
\hline
\end{tabular}

* Lethal-free homozygote $(\mathrm{S}-2 / \mathrm{S}-2)=1.000$

** Lethal-free heterozygote $(\mathrm{S}-2 / \mathrm{W}-109)=1.000$

\section{DISCUSSION}

Some lethal genes on the second chromosome of Drosophila melanogaster could persist in high frequency in experimental populations as observed in the case of PC 207 lethal gene $(l(2) 55 i)$, that is, $20-40 \%$ and about $20 \%$ in homozygous and heterozygous populations respectively. Allen $(1969,1970)$ stated that the level of frequency of heterotic lethal genes in natural populations might be underestimated with data of tests for fitness and allelism. However, these results are not so absolute that all persistent lethals in natural populations should show the heterosis in fitness. He also stated that some lethal loci can be balanced in heterozygous condition and that there is no general average effect with regard to lethal unless many conditions are specified, such as, genetic background, persistence in populations and other loci.

Oshima and Kitagawa (1961) tested the X-ray induced lethals on persistence in the population. Four out of six lethal heterozygotes had a relatively high coefficient of selection and other two lethal heterozygotes had negative coefficient of selection. The latter two lethal genes were maintained until the 10th generation in the experimental populations in which the frequencies were significantly higher than the theoretical one. They explained these results that the genetic background of flies in the population was presumed to be isogenic except for the irradiated second chromosome at the begining, but it might have become somewhat heterozygous by spontaneous mutations in the course of the following generations. The genetic background might be remained relatively homozygous until the 10th generation, therefore, the high frequency of lethal genes might be attributed to the superiority in preadult viability of their heterozygotes. The genetic background was proved to have a remarkable influence on the individual heterozygous effect of the lethal gene on viability.

Mukai and Burdick (1960) showed the particular lethal gene $l(2) 55 i$ on the second chromosome attained the equilibrium state in spite of different initial frequencies of the gene in the population. Persistence of a lethal gene in a population must be considered in relation to the degree of heterozygosity of genetic background as well as the state of coadaptation of chromosomes. Lethal genes which have been maintained at high frequency in a natural population experienced severe natural selection after their occur- 
rence. Therefore, some specific mechanisms of persistence of such genes might be considered. Oshima (1969) and Watanabe and Oshima (1970) discussed on the possible mechanisms of persistence of some lethal genes in natural populations. They showed that some mechanisms, such, as, linkage with an adaptive gene complex, heterotic inversions and segregation distorter gene (SD) on the basis of the distribution of these persistent lethal genes on the genetic map, might be involved.

From the present results, it was estimated that, in general, female fecundity of lethal heterozygotes carrying heterozygous genetic background was not so effective for keeping lethal genes at high level, though the fecundity value was assumed to be one of the important factors in fitness. There are many problems to be solved in order to clarify the mechanisms of persistence of lethal genes in natural and experimental populations, because their mechanisms would be different for each lethal gene.

\section{SUMMARY}

Female flies heterozygous for four kinds of recessive lethal genes on the second chromosome in homozygous genetic background showed high fecundity and the following results were ascertained by their persistence in the artificial populations.

1) Some lethals showed heterotic effect in homozygous genetic background.

2) Even these lethals which did not show high female fecundity in heterozygous background have been maintained at higher level than the theoretical one.

3) Decreasing patterns of each lethal heterozygote were similar in heterozygous and its irradiated genetic backgrounds.

4) The effect of irradiation gave rise to decrease the frequencies of lethal heterozygotes, but it was not clear in the case of heterozygous population.

However, the intimate relationship between female fecundity of lethal heterozygotes and equilibrium frequency of these lethal genes was presumed in the homozygous populations. So the fecundity was assumed to be an important factor in fitness and persisting mechanisms of lethals.

\section{ACKNOWLEDGMENTS}

The author would like to express his sincere thanks to Dr. C. Oshima, National Institute of Genetics, for his valuable discussions and criticism. He also thanks to Mrs. Mikiko (Fukasawa) Kondo and Miss Hatsue Tokuda for their technical assistance.

\section{LITERATURE CITED}

Allen, A., 1969 Lethal frequencies on second and third chromosomes in population of Drosophila melanogaster. Genetics 63: 629-637.

Allen, A., 1970 Lethal frequencies in laboratory population of D. melanogaster. Drosophila Inform Serv. 45: 104. 
Burdick, A. B., $1961 l(2) 55 i$ at Erie Pennsylvania. Drosophila Inform. Serv. 35: 75.

Mukai, T., and A. B. Burdick, 1960 Concerning equilibria of heterotic lethls in random mating population with particular reference to $l(2) 55 i$ in Drosophila melanogaster. Genetics 45: 1581-1593.

Oshima, C., and O. Kitagawa, 1961 Heterozygous effects of induced lethal genes on preadult viability in Drosophila melanogaster and their persistence in experimental populations. Japan. J. Genetics 36 Suppl.: 167-178.

Oshima, C., 1969 Persistence of some recessive lethal genes in natural populations of Drosophila melanogaster. Japan. J. Genetics 44 Suppl. 1: 209-216.

Spiess, E. B., R. B. Helling, and M. R. Capenos, 1963 Linkage of autosomal lethals from laboratory populations of Drosophila melanogaster. Genetics 48: 1377-1388.

Tano, S., and A. B. Burdick, 1965 Female fecundity of Drosophila melanogster second chromosome recessive lethal heterozygotes in homozygous and heterozygous genetic background. Genetics 51: 121-135.

Watanabe, T. K., and C. Oshima, 1970 Persistence of lethal genes in Japanese natural populations of Drosophila melanogaster. Genetics 64: 93-106.

Ytterborn, K. H., 1968 The persistency of experimental populations of second chromosome recessive lethals obtained after irradiation of spermatogonia and spermatozoa in Drosophila melanogaster. Hereditas 60: 33-71 